\title{
Probing surface quantum flows in deformed pygmy dipole modes
}

\author{
Wang, Kai
}

2017-09-06

Wang , K , Kortelainen, M \& Pei , J C 2017 , ' Probing surface quantum flows in deformed pygmy dipole modes ' , Physical Review C , vol. 96 , no. 3 , 031301 . https://doi.org/10.1103/PhysRevC.96.031301

http://hdl.handle.net/10138/233510

https://doi.org/10.1103/PhysRevC.96.031301

other

publishedVersion

Downloaded from Helda, University of Helsinki institutional repository.

This is an electronic reprint of the original article.

This reprint may differ from the original in pagination and typographic detail.

Please cite the original version. 


\title{
Probing surface quantum flows in deformed pygmy dipole modes
}

\author{
Kai Wang, ${ }^{1}$ M. Kortelainen, ${ }^{2,3}$ and J. C. Pei ${ }^{1, *}$ \\ ${ }^{1}$ State Key Laboratory of Nuclear Physics and Technology, School of Physics, Peking University, Beijing 100871, China \\ ${ }^{2}$ Department of Physics, P.O. Box 35 (YFL), University of Jyvaskyla, FI-40014 Jyvaskyla, Finland \\ ${ }^{3}$ Helsinki Institute of Physics, P.O. Box 64, University of Helsinki, FI-00014 Helsinki, Finland
}

(Received 11 December 2016; revised manuscript received 23 May 2017; published 6 September 2017)

\begin{abstract}
To explore the nature of collective modes in weakly bound nuclei, we have investigated deformation effects and surface flow patterns of isovector dipole modes in a shape-coexisting nucleus, ${ }^{40} \mathrm{Mg}$. The calculations were done in a fully self-consistent continuum finite-amplitude quasiparticle random phase approximation in a large deformed spatial mesh. An unexpected result of pygmy and giant dipole modes having disproportionate deformation splittings in strength functions was obtained. Furthermore, the transition current densities demonstrate that the long-sought core-halo oscillation in pygmy resonances is collective and compressional, corresponding to the lowest excitation energy and the simplest quantum flow topology. Our calculations show that surface flow patterns become more complicated as excitation energies increase.
\end{abstract}

\section{DOI: 10.1103/PhysRevC.96.031301}

Introduction. Atomic nuclei are evolving from few-body to many-body quantum systems and could exhibit amazing collective phenomena. In particular, when nuclei have large unbalanced neutron to proton ratios (i.e., a large isospin asymmetry) and associated significant charge-neutral surfaces, new coherent excitation modes are expected to appear $[1,2]$. The so-called "soft" or "pygmy" dipole resonance (PDR), corresponding to the relative oscillation between the core and the skin or halo from the hydrodynamical point of view [3], are especially intriguing. They are relevant to rich physics aspects such as the neutron skin and equation of state, as well as enhanced neutron capture rates in the astrophysical $r$-process [1]. Extensive experimental measurements have been performed to study the PDR, although mainly in less exotic nuclei $[2,4]$. Theoretically, however, it is of ultimate interest to explore the nature of pygmy resonances in weakly bound nuclei close to drip lines, in contrast to giant resonances.

It is expected that both pygmy and giant resonances can have nondegenerate modes induced by deformation effects. Since the deformed halo structures have been proposed [5-7], it has been desirable to identify decoupled halo-core shapes through comparative studies of anisotropic $K$ splittings in pygmy and giant dipole resonances (GDRs). However, detailed splitting behaviors of PDRs have not been studied yet. Furthermore, in PDRs of weakly bound nuclei, whether there exists a collective core-halo oscillation is still a long-standing question, which can be perceived directly via transition currents. The associated irrotational superfluid flow could be an intuitive PDR mode or a compressional mode or a toroidal mode, according to Refs. [8-10]. It is essential to study the flow patterns with broken spatial symmetries even for spherical nuclei, because by imposing symmetries the internal motions and flow patterns can be notably different [11]. The study of nuclear surface flow features should be of broad interest, considering the interesting flows in cold atomic gases [12] and quark matter [13].

\footnotetext{
*peij@pku.edu.cn
}

The suitable microscopic tool for the aforementioned studies is the fully self-consistent deformed continuum quasiparticle random phase approximation (QRPA), which takes into account weak-binding effects. The fully self-consistency is important for elimination of spurious states in order to obtain meaningful low-lying states. In addition, low-lying excitations can be sensitive to pairing and continuum effects $[14,15]$. Due to numerically challenging fully self-consistent treatment of the deformed continuum QRPA, the earlier studies have been usually limited to spherical symmetry $[14,15]$. Recently, the finite-amplitude-method QRPA (FAM-QRPA) has been proposed [16], which allows us to solve the QRPA problem iteratively, being more efficient compared to the conventional matrix-QRPA approach. In the present work, we demonstrate that the multipole excitations in deformed weakly bound nuclei can be studied with unprecedented numerical accuracy by adopting the FAM-QRPA approach in a large spatial mesh.

In this Rapid Communication, we aim to study deformation splittings and visualize the surface flow patterns in the PDR of the weakly bound deformed nucleus ${ }^{40} \mathrm{Mg}$. The calculations are done in the fully self-consistent, coordinate-space, deformed continuum FAM-QRPA framework. ${ }^{40} \mathrm{Mg}$ is the last experimentally observed magnesium isotope [17] with an $N=28$ magic neutron number, but with a well-established prolate-oblate shape coexistence $[18,19]$. We stress that such a shape-coexistence scenario is ideal to comparatively analyze deformation effects in pygmy resonances. Compared to earlier studies of ${ }^{40} \mathrm{Mg}$ [20], our calculations employ a large spatial mesh and, consequently, can provide details of a PDR which have never been revealed before.

Method. Here we describe the development of the FAMQRPA approach for multipole collective excitations based on Hartree-Fock-Bogoliubov (HFB) solutions in a large, axially symmetric coordinate-space mesh. In deformed weakly bound nuclei, there exists a subtle interplay among the surface deformation, surface diffuseness, and continuum coupling. Therefore, it is crucial to prepare very precise ground-state HFB solutions as a starting point for reliable descriptions of collective excitations. The HFB equation is solved with the computer code HFB-AX [21] within a large two-dimensional 
coordinate-space mesh, based on B-spline techniques for axially symmetric deformed nuclei [22]. For calculations employing large box sizes and small lattice spacings, the discretized continuum spectra would be very dense, providing good resolutions of quasiparticle resonances and continuum [7]. Note that the exact, fully self-consistent treatment of continuum in deformed cases [23] is rare and has not been used for QRPA calculations yet.

For the particle-hole interaction channel, a recently adjusted extended SLy4 force for light nuclei is adopted [24], including an additional density-dependent term. For the particle-particle channel, a density-dependent pairing interaction, $V_{0}[1-$ $\left.\eta\left[\rho(\boldsymbol{r}) / \rho_{0}(\boldsymbol{r})\right]^{\gamma}\right]$, is used [25]. With a pairing window of $60 \mathrm{MeV}$, the pairing force parameters are taken as $V_{0}=$ $-448.3 \mathrm{MeV} \mathrm{fm}^{3}, \eta=0.8$, and $\gamma=0.7$, so that pairing gaps in both stable and very neutron-rich nuclei can be properly described. The resulted pairing gaps are between those from mixed and surface types of pairing. A pure surface pairing interaction may overestimate pairing correlations in nuclei far from stability [26]. Detailed results with SLy4 and extendedSLy4 forces and different pairing interactions are shown in the Supplemental Material [27].

The next step is to combine the FAM-QRPA method with the deformed coordinate-space HFB approach. Recently, there have been a lot of new developments with the FAM-QRPA method [28]. In our previous work, we implemented FAMQRPA in axially deformed coordinate spaces for monopole transitions [29]. Very recently, the FAM-QRPA scheme with an arbitrary multipole operator has been realized [30].

In our FAM-QRPA calculations, to maintain full selfconsistency, the full quasiparticle basis and all time-odd terms are included. Note that the widely used canonical basis approach to apply truncations can undermine descriptions of halo tails [31]. To study the fine structures of pygmy resonances in the present work, an imaginary part of the excitation frequency $\omega$, for smoothing resonances, is taken to be $0.25 \mathrm{MeV}$, which is smaller than the usually adopted $0.5 \mathrm{MeV}$. The maximum angular momentum $z$-projection limit for quasiparticle states is taken as $\Omega_{\max }=39 / 2$. The storage of all wave functions takes about 17 gigabytes when a box size of $27.6 \mathrm{fm}$ is adopted. We solve the nonlinear multipole FAM-QRPA equation iteratively with the modified Broyden method. For each excitation frequency $\omega$ point, the calculation employs the OpenMP shared memory parallel scheme. For different frequencies, the MPI distributed parallel scheme is adopted. Consequently, the FAM-QRPA approach can be efficiently implemented by using this hybrid parallel scheme.

Results. We have performed calculations for the coexisting prolate and oblate shapes in ${ }^{40} \mathrm{Mg}$, which has a very soft potential energy surface [19]. The prolate shape $\left(\beta_{2}=0.39\right)$ is a weakly bound ground state with a neutron Fermi energy of $\lambda_{\mathrm{n}}=-0.33 \mathrm{MeV}$, which is a more reasonable value than the standard SLy4 result of $\lambda_{\mathrm{n}}=-0.52 \mathrm{MeV}$, considering that ${ }^{42} \mathrm{Mg}$ and ${ }^{39} \mathrm{Na}$ have not been experimentally observed yet. The oblate minimum $\left(\beta_{2}=-0.32\right)$ is energetically about $1.9 \mathrm{MeV}$ above the prolate minimum, but has a lower Fermi energy of $\lambda_{\mathrm{n}}=-0.79 \mathrm{MeV}$. Note that the isotone ${ }^{42} \mathrm{Si}$ has a welldeformed oblate ground state [32]. This shape competition is also reflected in superfluid properties. The prolate shape has a

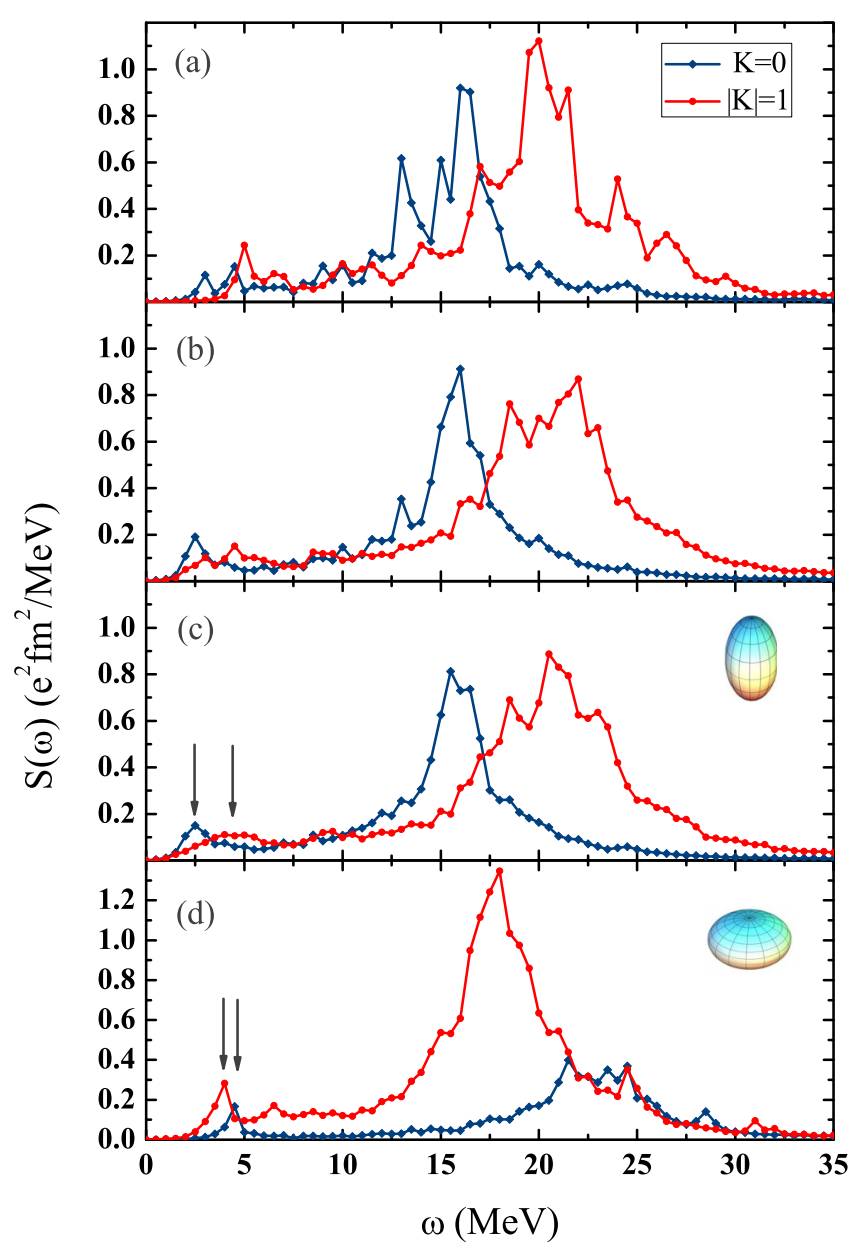

FIG. 1. Transition strength functions of isovector dipole resonances in the shape-coexisting nucleus ${ }^{40} \mathrm{Mg}$ as a function of excitation energy, calculated with different box sizes. (a) Prolate shape with a box size of $12 \mathrm{fm}$; (b) prolate shape with a box size of $21 \mathrm{fm}$; (c) prolate shape with a box size of $27.6 \mathrm{fm}$; (d) oblate shape with a box size of $27.6 \mathrm{fm}$.

neutron pairing gap of $\Delta_{\mathrm{n}}=1.23 \mathrm{MeV}$ (while $\Delta_{\mathrm{p}}=0$ ), and the oblate shape has a proton pairing gap of $\Delta_{\mathrm{p}}=0.98 \mathrm{MeV}$ (while $\Delta_{\mathrm{n}}=0$ ).

The electric isovector dipole resonances at the prolate and oblate local energy minima can be self-consistently obtained without spurious states, since, within FAM-QRPA, the isovector dipole operator does not excite spurious center-of-mass modes. The isovector PDR is a natural probe of relative surface oscillations and can be also related to the nuclear photoabsorption cross section. The isoscalar PDR, which suffers from spurious states [33,34], is not investigated presently. In Fig. 1, the calculated FAM-QRPA transition strength functions of $K=0$ and $|K|=1$ (with sum of $K=1$ and $K=-1$ ) cases are shown. Our implementation has been benchmarked with the calculation of ${ }^{24} \mathrm{Mg}$ (see details in Ref. [27]). It is known that soft monopole resonances are mainly due to continuum effects rather than to single-particle structures [29], and this also holds for soft multipole resonances. To see the role of the accurate treatment of continuum and halo extensions, the 
transition strengths have been calculated with box sizes of 12,21 , and $27.6 \mathrm{fm}$. We can see that within a small box, the continuum discretization is insufficient and several false peaks appear. Even with a box size of $21 \mathrm{fm}$, a false peak at $13 \mathrm{MeV}$ is present. Moreover, it can be seen that pygmy resonances are fragmented and less coherent with smaller box sizes.

With calculations employing a large box size of $27.6 \mathrm{fm}$, the obtained transition strengths clearly demonstrate smoothed pygmy resonances and deformation-induced splittings, as shown in Figs. 1(c) and 1(d). It is known that the anisotropic splitting in the dipole transition strength is approximately proportional to the centroid excitation energy and the deformation, based on a hydrodynamic model [35]. The microscopic RPA calculations have confirmed that the GDR splitting depends linearly on the deformation [36]. The results show that prolate and oblate shapes have similar GDR splittings $\left(\delta_{E} \sim 5 \mathrm{MeV}\right)$. Considering different centroid energies, the estimated PDR deformation splittings should be around $0.95 \mathrm{MeV}$ for the prolate shape and $1.05 \mathrm{MeV}$ for the oblate shape. However, we see the PDR deformation splitting of the prolate shape $\left(\delta_{E}=1.4 \mathrm{MeV}\right)$ is significantly larger than the expected value whereas the oblate case $\left(\delta_{E}=0.45 \mathrm{MeV}\right)$ is on the contrary. Calculated density distributions show no core-halo shape decoupling in ${ }^{40} \mathrm{Mg}$ (see [27]). Obviously, the mechanism for the PDR deformation splitting differs from the GDR splitting. From tests, we have found that the obtained deformation splittings were not sensitive to pairing gaps. Therefore, we speculate that the pygmy deformation splitting is related to not only the static shape but also significant dynamical surface effects. It will be very helpful to look for the PDR deformation splitting by high-resolution experimental measurements of deformed neutron-rich nuclei. The dominating $|K|=1$ mode in the oblate case and also the total cross section differ notably from the prolate case.

To understand the different collective natures between pygmy and giant resonances, we have further studied the transition density $\delta \rho$ and the transition current density $\delta \vec{j}$ at resonance peaks. Note that the smoothing of resonances has been included in solving the FAM-QRPA equation. Figure 2 displays the imaginary part of transition density distributions of $K=0$ modes of the prolate shape. We see that in the pygmy resonance the neutron-proton transition densities have basically an in-phase pattern, whereas the giant resonance has a characteristic pattern of opposite phases. This is consistent with the relative core-halo motion in pygmy resonances. This in-phase pattern has been pointed out in the literature and is related to strong isoscalar PDR, e.g., in Refs. [20,37,38]. However, a contrary interpretation was obtained with RPA calculations in Ref. [39]. For the weakly bound ${ }^{40} \mathrm{Mg}$, we see the neutron transition density has a much larger spatial extension than that of protons. The excessive neutron surface is the main source of the PDR transition strength.

Figure 3 displays the imaginary part of the neutron transition current density $\delta \vec{j}$ on a cylinder $(r, z)$ plane with azimuthal angle $\phi=0$. The transition current density can be used as a direct probe of the collective nature of pygmy resonances. The very small current fields at surfaces actually correspond to considerable large velocity fields [27]. To compare with the superfluid prolate case, the pairing strength in calculations of

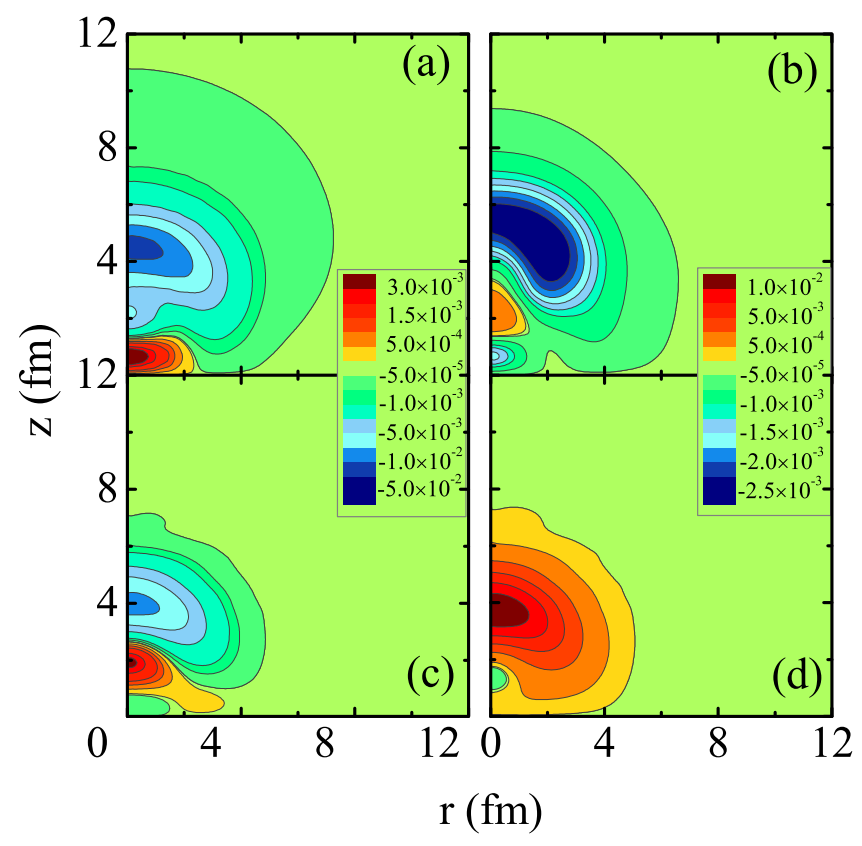

FIG. 2. $K=0$ transition density distributions of pygmy and giant dipole resonances in ${ }^{40} \mathrm{Mg}$ with the prolate shape displayed in the cylinder coordinate space as $\delta \rho(r, z)$ for (a) neutrons of PDR, (b) neutrons of GDR, (c) protons of PDR, and (d) protons of GDR.

oblate current flows has been increased by $5 \%$. This does not affect the resulting PDR deformation splitting.

In Fig. 3(a), the current flow of the prolate shape $K=0$ PDR illustrates a very clear compressional pygmy mode,

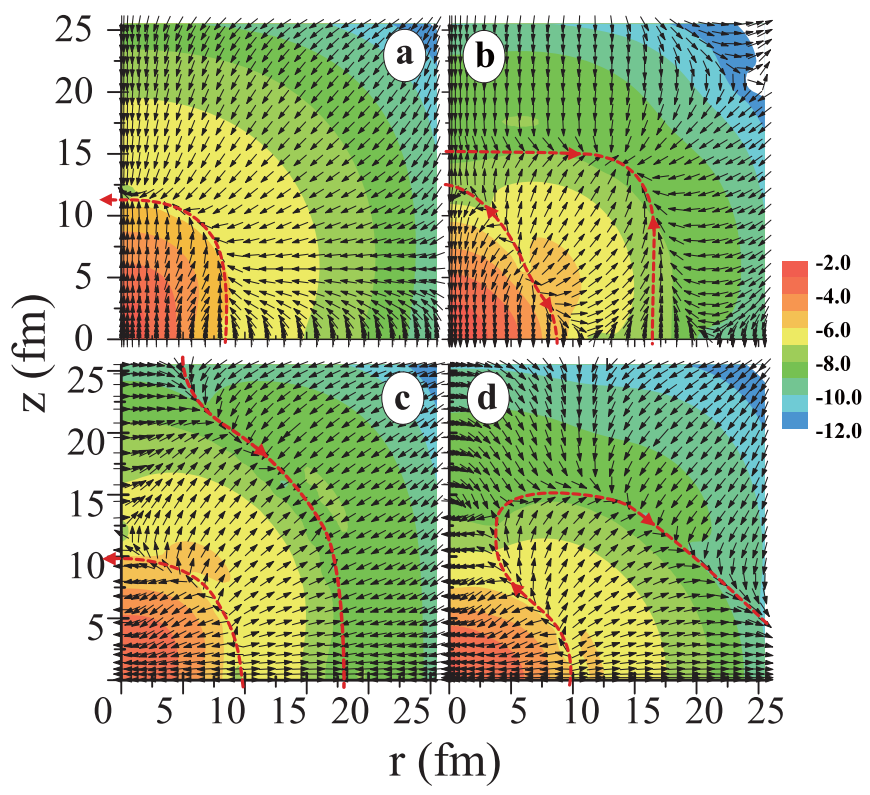

FIG. 3. Neutron transition current density in the cylinder coordinate space, $\delta \vec{j}(r, z, \phi=0)$, of the PDR in ${ }^{40} \mathrm{Mg}$. The color scales denote the logarithm of the current strength and the arrows denote the flow direction. (a) $K=0$ mode of the prolate shape, (b) $K=0$ mode of the oblate shape, (c) $K=1$ mode of the prolate shape, and (d) $K=1$ mode of the oblate shape. The flow boundary lines are given for guiding eyes. 


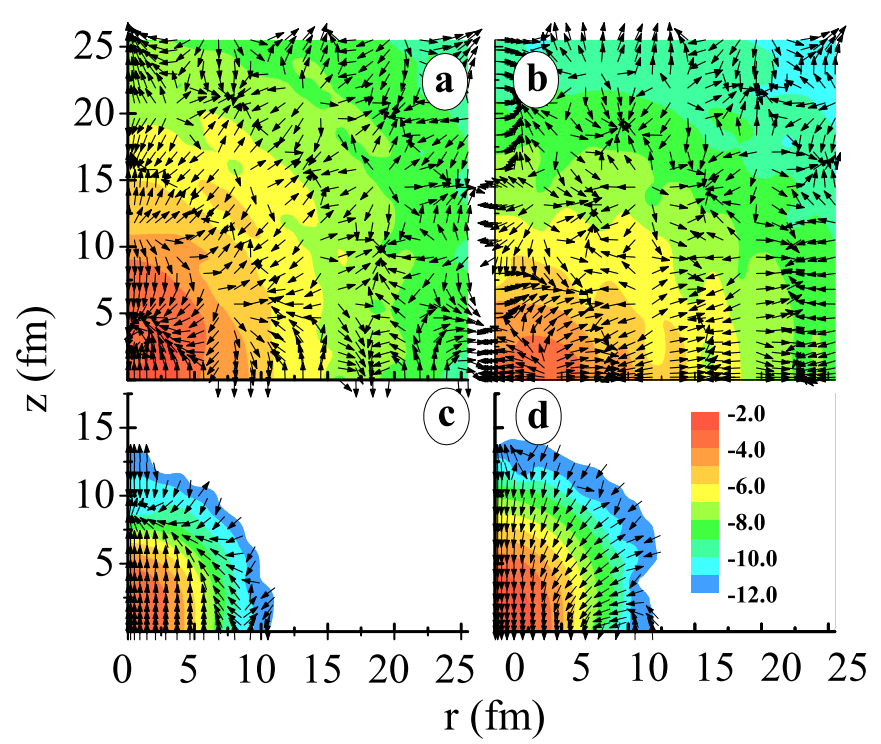

FIG. 4. Transition currents similar to Fig. 3, but for (a) the neutron $K=0$ prolate GDR, (b) the neutron $K=1$ oblate GDR, (c) the proton $K=0$ prolate PDR, and (d) the proton $K=0$ prolate GDR.

which is exactly the long-sought collective surface-core oscillation [9]. There is a flow-in pole at $z=12 \mathrm{fm}$ along the $z$ axis, connected with the corresponding flow-out pole at $z=-12 \mathrm{fm}$ due to the reflection symmetry, showing a typical dipole structure. The boundary between centripetal inward and outward flows appears at a distance of $12 \mathrm{fm}$ from the center. Such a large boundary distance is related to a very soft excitation $(2.5 \mathrm{MeV})$. The $K=0 \mathrm{PDR}$ of the prolate shape has the simplest flow topology, associated with the lowest energy $(2.5 \mathrm{MeV})$. On the other hand, the flows corresponding to Figs. 3(b)-3(d) all have two boundaries with a rebound wave and have similar excitation energies $(4 \sim 4.5 \mathrm{MeV})$. This energy dependence of flow patterns may be related to the disproportionate $K$ splittings in PDR as shown in Fig. 1. We see the compressional flow patterns are characterized by boundary lines, in analog to the topological winding numbers, but without any internal vorticity. Calculations with different box sizes up to $34 \mathrm{fm}$ have been performed [27], which confirm that the compressional PDR pattern is a collective quantum phenomenon, independent of box sizes. The dominance of surface compressional flows in weakly bound nuclei is not a surprise since the dilute nuclear surface has a very small incompressibility and, consequently, a remarkable soft monopole mode [29].

It is worth mentioning the flow pattern of the $K=1 \mathrm{PDR}$ of the oblate shape in Fig. 3(d). We see that two boundaries are connected and a flow circulation is generated. There have been several studies [8-10] pointing out the possible existence of a toroidal mode in neutron-rich nuclei, which may be favorable in the isoscalar PDR at higher energies. In our results, with the standard isovector dipole operator, the flow circulation is a synthetic pattern from sideward compressional flows (not an explicit toroidal mode), demonstrating the complexity of flow patterns due to the finite-size deformation effect and the possible interplay with the toroidal mode. By contrast, this circulation is not seen in the centripetal $K=1$ PDR mode of the prolate shape.

In Figs. 4(a) and 4(b), the neutron surface current flows of the $K=0$ prolate and $K=1$ oblate GDR are much more complex than the PDR case. Nevertheless, the flow pattern of the $K=0$ prolate GDR has several compressional ring structures. The compressional structures in GDR have been demonstrated again by calculations with different box sizes [27]. In addition, there is an orderly displacement wave at box boundaries. There are some flow poles and disorders as a consequence of flow interferences, depending on box sizes. As the excitation energy increases, distortions in the nuclear inner part appear in the flow. Generally, it is difficult to identify geometrical shape effects in flow patterns in giant resonances.

Figures 4(c) and 4(d) show the proton flows of the prolate pygmy and giant resonances. The proton flows have much smaller spatial extensions and show no distortions in either case. The in-phase flow pattern of PDR is very evident as shown in Figs. 3(a) and 4(c). A possible way to investigate the charge-neutral currents at outer nuclear surfaces, connected to novel scissors and twist modes, is through magnetic transitions [40].

Summary. With the newly developed fully self-consistent continuum deformed FAM-QRPA approach in a large spatial mesh, we have investigated the distinct collective nature of pygmy and giant dipole resonances of ${ }^{40} \mathrm{Mg}$ associated with weak-binding effects. First, the deformation splitting in pygmy resonances was found to be disproportional to the GDR splitting. This disproportion is not due to the static core-halo shape decoupling effect, implying considerable dynamical surface effects at low energies. Furthermore, the transition current flows illustrate very different topologies associated with excitation energies and geometrical shapes in pygmy resonances. The long-sought surface-core PDR oscillation is collective and compressional, corresponding to the simplest quantum flow topology, which occurs at a large distance boundary as the lowest $K=0$ mode of the ground-state prolate shape. The flow pattern has been demonstrated to be a robust quantum phenomenon by calculations with different box sizes. The surface flow patterns were seen to become more complicated as excitation energies increase. The analysis of these transition current flows can provide insight into the pygmy resonance, in addition to the analysis of transition densities. The present study demonstrates that anisotropic large coordinate-space calculations are essential for exploring soft excitations in weakly bound nuclei.

Acknowledgments. Useful comments by W. Nazarewicz and F. R. Xu are gratefully acknowledged. This work was supported by the National Natural Science Foundation of China under Grants No. 11522538, No. 11375016, and No.11235001. This work was also supported (M.K.) by the Academy of Finland under the Centre of Excellence Programme 2012-2017 (Nuclear and Accelerator Based Physics Programme at JYFL) and FIDIPRO program. We also acknowledge that computations in this work were performed in Tianhe-1A located at Tianjin and Tianhe-2 located at Guangzhou. 
[1] N. Paar, D. Vretenar, E. Khan, and G. Colò, Rep. Prog. Phys 70, 691 (2007).

[2] D. Savran, T. Aumann, and A. Zilges, Prog. Part. Nucl. Phys. 70, 210 (2013).

[3] R. Mohan, M. Danos, and L. C. Biedenharn, Phys. Rev. C 3, 1740 (1971).

[4] N. Ryezayeva, T. Hartmann, Y. Kalmykov, H. Lenske, P. von N.-Cosel, V. Y. Ponomarev, A. Richter, A. Shevchenko, S. Volz, and J. Wambach, Phys. Rev. Lett. 89, 272502 (2002); T. Hartmann, M. Babilon, S. Kamerdzhiev, E. Litvinova, D. Savran, S. Volz, and A. Zilges, ibid. 93, 192501 (2004); P. Adrich, A. Klimkiewicz, M. Fallot, K. Boretzky, T. Aumann, D. Cortina-Gil, U. DattaPramanik, T. W. Elze, H. Emling, H. Geissel, M. Hellstrom, K. L. Jones, J. V. Kratz, R. Kulessa, Y. Leifels, C. Nociforo, R. Palit, H. Simon, G. Surowka, K. Summerer, and W. Walus, ibid. 95, 132501 (2005); D. Savran, M. Babilon, A. M. vandenBerg, M. N. Harakeh, J. Hasper, A. Matic, H. J. Wortche, and A. Zilges, ibid. 97, 172502 (2006); J. Gibelin, D. Beaumel, T. Motobayashi, Y. Blumenfeld, N. Aoi, H. Baba, Z. Elekes, S. Fortier, N. Frascaria, N. Fukuda, T. Gomi, K. Ishikawa, Y. Kondo, T. Kubo, V. Lima, T. Nakamura, A. Saito, Y. Satou, J. A. Scarpaci, E. Takeshita, S. Takeuchi, T. Teranishi, Y. Togano, A. M. Vinodkumar, Y. Yanagisawa, and K. Yoshida, ibid. 101, 212503 (2008); A. P. Tonchev, S. L. Hammond, J. H. Kelley, E. Kwan, H. Lenske, G. Rusev, W. Tornow, and N. Tsoneva, ibid. 104, 072501 (2010); D. M. Rossi, P. Adrich, F. Aksouh, H. Alvarez-Pol, T. Aumann, J. Benlliure, M. Bohmer, K. Boretzky, E. Casarejos, M. Chartier, A. Chatillon, D. Cortina-Gil, U. DattaPramanik, H. Emling, O. Ershova, B. Fernandez-Dominguez, H. Geissel, M. Gorska, M. Heil, H. T. Johansson, A. Junghans, A. Kelic-Heil, O. Kiselev, A. Klimkiewicz, J. V. Kratz, R. Krucken, N. Kurz, M. Labiche, T. LeBleis, R. Lemmon, Y. A. Litvinov, K. Mahata, P. Maierbeck, A. Movsesyan, T. Nilsson, C. Nociforo, R. Palit, S. Paschalis, R. Plag, R. Reifarth, D. Savran, H. Scheit, H. Simon, K. Summerer, A. Wagner, W. Walus, H. Weick, and M. Winkler, ibid. 111, 242503 (2013); R. Massarczyk, R. Schwengner, F. Donau, S. Frauendorf, M. Anders, D. Bemmerer, R. Beyer, C. Bhatia, E. Birgersson, M. Butterling, Z. Elekes, A. Ferrari, M. E. Gooden, R. Hannaske, A. R. Junghans, M. Kempe, J. H. Kelley, T. Kogler, A. Matic, M. L. Menzel, S. Muller, T. P. Reinhardt, M. Roder, G. Rusev, K. D. Schilling, K. Schmidt, G. Schramm, A. P. Tonchev, W. Tornow, and A. Wagner, ibid. 112, 072501 (2014).

[5] T. Misu, W. Nazarewicz, and S. Aberg, Nucl. Phys. A 614, 44 (1997).

[6] S. G. Zhou, J. Meng, P. Ring, and E. G. Zhao, Phys. Rev. C 82, 011301(R) (2010).

[7] J. C. Pei, Y. N. Zhang, and F. R. Xu, Phys. Rev. C 87, 051302(R) (2013); Y. N. Zhang, J. C. Pei, and F. R. Xu, ibid. 88, 054305 (2013).

[8] D. Vretenar, N. Paar, P. Ring, and T. Nikšić, Phys. Rev. C 65, 021301(R) (2002).

[9] A. Repko, P.-G. Reinhard, V. O. Nesterenko, and J. Kvasil, Phys. Rev. C 87, 024305 (2013).

[10] P. Papakonstantinou, J. Wambach, E. Mavrommatis, and V. Y. Ponomarev, Phys. Lett. B 604, 157 (2004).

[11] P. Ring and P. Schuck, The Nuclear Many-Body Problem (Springer, Berlin, 1980).

[12] A. Bulgac, Y.-L. Luo, P. Magierski, K. J. Roche, and Y. Yu, Science 332, 1288 (2011).
[13] C. L. Zhou, Y. G. Ma, D. Q. Fang, G. Q. Zhang, J. Xu, X. G. Cao, and W. Q. Shen, Phys. Rev. C 90, 057601 (2014).

[14] M. Matsuo, Nucl. Phys. A 696, 371 (2001).

[15] E. Khan, N. Sandulescu, M. Grasso, and N. V. Giai, Phys. Rev. C 66, 024309 (2002).

[16] P. Avogadro and T. Nakatsukasa, Phys. Rev. C 84, 014314 (2011).

[17] T. Baumann et al., Nature 449, 1022 (2007).

[18] R. Rodríguez-Guzmán, J. L. Egido, and L. M. Robledo, Phys. Rev. C 65, 024304 (2002).

[19] J. Terasaki, H. Flocard, P-H. Heenen, and P. Bonche, Nucl. Phys. A 621, 706 (1997).

[20] K. Yoshida, Phys. Rev. C 80, 044324 (2009).

[21] J. C. Pei, M. V. Stoitsov, G. I. Fann, W. Nazarewicz, N. Schunck, and F. R. Xu, Phys. Rev. C 78, 064306 (2008).

[22] E. Terán, V. E. Oberacker, and A. S. Umar, Phys. Rev. C 67, 064314 (2003).

[23] H. Oba and M. Matsuo, Phys. Rev. C 80, 024301 (2009).

[24] X. Y. Xiong, J. C. Pei, and W. J. Chen, Phys. Rev. C 93, 024311 (2016).

[25] A. Pastore, F. Barranco, R. A. Broglia, and E. Vigezzi, Phys. Rev. C 78, 024315 (2008).

[26] S. A. Changizi and C. Qi, Phys. Rev. C 91, 024305 (2015).

[27] See Supplemental Material at http://link.aps.org/supplemental/ 10.1103/PhysRevC.96.031301 for the benchmark of our FAMQRPA codes, the comparision between SLy4 and extended SLy4 forces, systematic studies of different pairing interactions, ground-state density distributions, and the obtained transition flows of PDRs and GDRs in different box sizes.

[28] M. Stoitsov, M. Kortelainen, T. Nakatsukasa, C. Losa, and W. Nazarewicz, Phys. Rev. C 84, 041305(R) (2011); T. Nikšić, N. Kralj, T. Tutiš, D. Vretenar, and P. Ring, ibid. 88, 044327 (2013); H. Liang, T. Nakatsukasa, Z. Niu, and J. Meng, ibid. 87, 054310 (2013); N. Hinohara, M. Kortelainen, and W. Nazarewicz, ibid. 87, 064309 (2013); M. T. Mustonen, T. Shafer, Z. Zenginerler, and J. Engel, ibid. 90, 024308 (2014).

[29] J. C. Pei, M. Kortelainen, Y. N. Zhang, and F. R. Xu, Phys. Rev. C 90, 051304(R) (2014).

[30] M. Kortelainen, N. Hinohara, and W. Nazarewicz, Phys. Rev. C 92, 051302(R) (2015); T. Oishi, M. Kortelainen, and N. Hinohara, ibid. 93, 034329 (2016).

[31] J. Dobaczewski, W. Nazarewicz, T. R. Werner, J. F. Berger, C. R. Chinn, and J. Dechargé, Phys. Rev. C 53, 2809 (1996).

[32] S. Takeuchi, M. Matsushita, N. Aoi, P. Doornenbal, K. Li, T. Motobayashi, H. Scheit, D. Steppenbeck, H. Wang, H. Baba, D. Bazin, L. Caceres, H. Crawford, P. Fallon, R. Gernhauser, J. Gibelin, S. Go, S. Grevy, C. Hinke, C. R. Hoffman, R. Hughes, E. Ideguchi, D. Jenkins, N. Kobayashi, Y. Kondo, R. Krucken, T. LeBleis, J. Lee, G. Lee, A. Matta, S. Michimasa, T. Nakamura, S. Ota, M. Petri, T. Sako, H. Sakurai, S. Shimoura, K. Steiger, K. Takahashi, M. Takechi, Y. Togano, R. Winkler, and K. Yoneda, Phys. Rev. Lett. 109, 182501 (2012).

[33] J. Terasaki, J. Engel, M. Bender, J. Dobaczewski, W. Nazarewicz, and M. Stoitsov, Phys. Rev. C 71, 034310 (2005).

[34] D. P. Arteaga and P. Ring, Phys. Rev. C 77, 034317 (2008).

[35] G. F. Bertsch and R. A. Broglia, Oscillations in Finite Quantum Systems (Cambridge University, New York, 1994).

[36] D. P. Arteaga and E. Khan, and P. Ring, Phys. Rev. C 79, 034311 (2009). 
[37] N. Paar, D. Vretenar, and P. Ring, Phys. Rev. Lett. 94, 182501 (2005).

[38] N. Paar, Y. F. Niu, D. Vretenar, and J. Meng, Phys. Rev. Lett. 103, 032502 (2009).
[39] P.-G. Reinhard and W. Nazarewicz, Phys. Rev. C 87, 014324 (2013).

[40] K. Heyde, P. von N.-Cosel, and A. Richter, Rev. Mod. Phys. 82, 2365 (2010). 